\title{
Making light-spots travel further
}

\section{Careful shaping of the profile of a light beam makes it possible to increase greatly the distance over which the peak intensity is not degraded by diffraction.}

$\mathrm{ON}_{\mathrm{N}}$ the face of things, as schoolboys know. it is a kind of nonsense to think that it may be possible to arrange for a beam of light whose physical dimensions are finite to propagate through space without becoming broader and more diffuse because of diffraction. The usual and simplest explanation is based on Huygens's principle: each point on the instantaneous wavefront of a propagating beam can be thought of as the source of an outwardly propagating shell of radiation. For radiation propagating outwards from a point source, the wavefronts are spherical, and a sequence of ever-larger spherical wavefronts is obtained by the mutual interference of the hypothetical spherical wavelets travelling outwards from neighbouring points on each spherical surface. In the days when the lumeniferous aether was all the rage, this simple picture was one way of relating the supposed elastic properties of the aether to such quantities as the velocity of light and the electrodynamic constants of the vacuum. The same result obtains for plane waves travelling in one direction which are infinite in extent in the planes perpendicular to the direction of travel. All that is elementary. Equally. it is readily imagined that a plane wave which is not infinite in its lateral extent cannot travel without spreading. The Huygens wavelets near the middle of each wave front may interfere destructively except along another plane surface displaced slightly downstream, but the Huygens wavelets spreading from near the edges of the wavefront will not be neatly cancelled out by their neighbours except along the surface of the displaced plane, so that the travelling beam becomes fuzzy at the edges.

Of necessity, the spreading-out obtains simply as a consequence of the finite size of the propagating beam, and occurs even in a vacuum. The distance over which a beam of circular cross-section $r$ will remain reasonably compact is measured by its cross-sectional area divided by the wavelength (which is why those who would use lasers in defences against ballistic missiles must think of building instruments with very large cross-section).

But now, it seems, there is a way round the problem. J. Durnin of the Institute of Optics at the University of Rochester has apparently managed to find a solution of the wave equation which represents a beam of light of finite cross-section which is immune from this diffraction phenomenon. Moreover, Durnin and two colleagues at the same university, J.J. Miceli and J.H. Eberly, have been able to construct in the laboratory a realization of Durnin's wave (Phys. Rev. Lett. 58, 1499; 1987). Maddeningly, Durnin's account of his discovery, which will no doubt eventually explain how he came by his solution, is cited as "in the press with $J$. Opt. Soc. Am." (Physicists - and physics journals -are becoming terribly cavalier in this respect, chiefly under the influence of their huge trade in preprints. It seems nowadays quite common that articles based on pre-prints appear in print before the originals that inspired them. It is more unusual that an experiment to confirm the correctness of a theory should appear in advance of the explanation. But either way, the practice is confusing.)

Not unexpectedly, the beam is really rather special. The intensity is far from uniform across the cross-section of the beam but, rather, consists of a relatively intense central spot surrounded by an infinite series of annular rings of light whose intensity is inversely proportional to their radius (measured from the central spot). The fact that this description may be reminiscent of the diffraction plates by means of which the high-definition Schmidt sky-cameras are constructed is far from coincidental; in both cases, the trick is a solution of the wave equation which, in cylindrical coordinates, represents a beam travelling along the axial direction $z$; the solution of interest is then the product of an oscillatory function of $z$ and the Bessel function of the first kind of zeroth order, known in the trade as $J_{0}(a r)$, where $a$ is a constant and $r$ is the off-axis distance.

The most striking feature of this beam profile is that it represents a sharp central peak surrounded by a sequence of oscillations which tail off to zero inversely with the distance. The half-width of the central spot is inversely proportional to the constant $a$. It simply requires a little differentiation to show that the function is indeed a solution of the wave equation. But the fact that the oscillatory function of $z$ is multiplied in the solution by a function of the radius only shows that the profile of the beam is literally unchanged on propagation, at least so long as the space in which the wave is travelling is for practical purposes infinite.
How to realize this state of affairs in the laboratory? What Durdin and his colleagues have done is to place an annular slit illuminated by well-collimated light in the focal plane of a lens of comparable dimensions. The slit must not be so narrow as to cause diffraction effects on its own account nor so wide as to confuse the lens. The output from the lens is a conical band of light. Direct measurement shows that the on-axis beam will propagate for roughly $1 \mathrm{~m}$ (under the conditions of the experiment) without substantial degradation of the central spot (whereafter it quickly falls to zero). Direct measurement has shown that a comparable spot-like beam with a gaussian profile decreases in intensity by an order of magnitude in a few centimetres.

The immediate application of this development is in those circumstances in which it is sought to make laser beams travel vast distances with undiminished intensity. It is worth noting that a perfectly collimated wave from a $2-\mathrm{cm}$ diameter laser will have lost much of its central intensity after travelling for $1 \mathrm{~km}$ in a vacuum. Because the distance increases with the square of the radius, the modestly larger instruments have markedly better performance, but if it is in practice possible to enhance the distance over which the central intensity is not degraded by something like a factor of 100 by an appropriate shaping of the beam, many now impractical tasks will be possible (or present jobs can be done with smaller instruments). Curiously enough, there is no reference to the Strategic Defense Initiative among the references.

One curious problem remains. One of the other lessons learned at school is that the diffraction of a beam of finite crosssection is not merely an annoying consequence of Huygens' principle but a consequence of Heisenberg's uncertainty principle. But Durnin and his colleagues appear to have improved on that by a factor of a hundred or thereabouts. What can be the explanation? The authors provide the answer off their own bat: the explanation is that, although the central and practically important feature of the Bessel beam is its narrow central spot, the outlying annular rings of light intensity imply that the beam is, for practical purposes, infinite in its lateral dimensions. Both Huygens and Heisenberg survive. 